\title{
Reaction Systems for Modeling and Validation of Biological Signaling Pathways: G1/S Checkpoint of the Cell Cycle
}

\author{
Nimet İlke Akçay ${ }^{1}$, Benedek Nagy ${ }^{2, *}$, Şükrü Tüzmen ${ }^{3}$ \\ ${ }^{1}$ Faculty of Medicine, Eastern Mediterranean University, Famagusta, North \\ Cyprus, Mersin-10, Turkey, ilke.cetin@emu.edu.tr \\ ${ }^{2}$ Department of Applied Mathematics and Computer Science, Eastern \\ Mediterranean University, Famagusta, North Cyprus, Mersin-10, Turkey, \\ benedek.nagy@emu.edu.tr \\ ${ }^{3}$ Faculty of Dentistry, Eastern Mediterranean University, Famagusta, North \\ Cyprus, Mersin-10, Turkey, sukru.tuzmen@emu.edu.tr \\ * Corresponding author
}

Abstract: Reaction Systems modeling is a compact, discrete, and qualitative modeling method for dynamic systems, including biochemical systems. In this paper, the G1/S checkpoint of the human cell cycle is modeled by Reaction Systems. This specific checkpoint is important since it plays role in tumor formation. Modeling discrete dynamic systems with Reaction Systems has many advantages over other modeling tools, i.e., the model is very small, deterministic and it is easy to use in simulations without any specific software package.

Keywords: Cell Cycle; G1/S checkpoint; Reaction Systems; Simulation

\section{Introduction}

The cell cycle is a sequence of events, which occurs in a cell leading to its proliferation and division in order to give rise to two identical daughter cells. For a healthy being, correct regulation is required to avoid any cell aberrancy. Should there be any aberrations along this sequence of events, there will be disruptions in the orchestration of the downstream events, which may lead to genetic disorders, such as cancer. Interdisciplinary scientific research has gained impetus for the past two decades. The application of a variety of mathematical tools and techniques to model biological and biomedical systems have facilitated qualitative and quantitative description, simulation, and prediction of events that may not be 
otherwise evident to the researcher. The marrying of the two disciplines, mathematics and biology, has been greatly facilitated by the accumulated data-rich genomic information sets, which are difficult to comprehend without the utilization of analytical tools; up-to-date mathematical tools which make understand complex, nonlinear biological systems easy; increasing computing power that can ease calculations and simulations that were not previously feasible, and growing interest in in silico experiments about human and animal research. Consequently, the interaction between two disciplines facilitates the interpretation of complex biological data.

In studies for modeling and simulating biological systems; different modeling approaches have been applied so far, including Process Algebra, Rule-based Systems, Petri Nets, Boolean Networks, State Charts and Hybrid Systems [1].

The eukaryotic cell cycle is a well-studied, extremely complex phenomenon, which leads to cancer upon its misregulation. This system exhibits a good example of a mathematical model presenting interpretable results. By utilizing the values and mode of interaction of different parameters, we can be able to demonstrate the changes in the modules of cell cycle events. Within the last decades, many mathematical models of the cell cycle or its specific checkpoints have been constructed. Although majority of these models were constructed by the use of Ordinary Differential Equations [2, 3, 4, 5]; Petri Net models were also constructed with hybrid $[6,7,8,9]$ and stochastic approaches [10].

Reaction Systems (RSs) were invented [11, 12] to simulate biochemical reactions. In order to reflect the behavior of biochemical systems, RSs contain a set of objects, e.g., chemicals, and a collection of reactions. Each reaction has its own set of reactants, inhibitors, and products. Reactions are enabled according to conditions given by their sets of reactants and inhibitors, and enabled reactions produce their products. An RS is deterministic, thus simulations started from the same initial sets result in the same products.

Many theoretical studies have been published so far, including extensions of RSs, functions or sequences generated by RSs, and programming frameworks. In addition, the studies on applications of RSs to model biological networks are being accumulated; such as RSs model for the heat shock response [13], simulating gene regulatory networks using RSs [14], and simulating potential therapeutic modalities to reawaken fetal hemoglobin [15].

The basic RS that we have adapted for our case, the G1/S checkpoint of the cell cycle, is finite. Hence, all computational problems regarding this case can be easily solved via simulations. 


\section{Biological Context}

\subsection{The Cell Cycle}

The cell cycle [16] consists of four ordered and irreversible phases: G1, S, G2, and M. G1 phase is the first gap period in which cells decide whether to proliferate or not based on the information received from the extracellular environment. If proliferation is achieved, the cells grow to reach twice their size in the G1 phase. In the $\mathrm{S}$ phase, DNA is duplicated to produce two identical daughter cells. During the G2 phase, cell growth and protein synthesis continue to occur until the preparation for mitosis is completed. Mitosis (M) is the phase in which a cell is divided into two identical daughter cells by following the stages prophase, metaphase, anaphase, and telophase [17, 18]. Errors during the cell cycle induce either apoptosis (known as programmed cell death) [19, 20, 21] or cause mutations. If cell division is completed with such mutations, or the mechanism of apoptosis is damaged, then there is a high possibility of cancer to emerge. In order to control and fix such damages before the cell cycle is completed; cyclins, cyclindependent kinases (CDKs), and cyclin-dependent kinase inhibitors (CKIs) play key roles $[22,23]$.

\subsection{Cyclins, CDKs and CKIs}

Cyclins are proteins that have roles to control various phases of the cell cycle. They are substrates for CDKs that regulate the cell cycle by phosphorylating (adding phosphate molecules to) other proteins. CDKs are not activated unless they bind to associated cyclins.

CKIs are another class of proteins that regulate the kinase activity of Cyclin-CDK complexes. Under unfavorable conditions such as DNA damage, abnormalities during the cell cycle, or environmental stresses, CKIs bind to Cyclin-CDK complexes to inhibit the cell cycle progression until the damage is under control.

\subsection{Cell Cycle Checkpoints}

DNA damage is prone to happen especially on proliferating cells. Cell cycle checkpoints are an array of mechanisms to control DNA damage occurring during the cell cycle and sustain the division process until the damage is repaired. Each checkpoint can be represented by complex signaling pathways in which a various number of genes and proteins play significant roles. There are three checkpoints throughout the cell cycle [24]: G1/S checkpoint [25, 26], G2/M checkpoint [27, 28], and M phase spindle checkpoint [24, 29, 30]. 


\subsubsection{G1/S Checkpoint}

In this study, our focus is on the G1/S checkpoint $[25,26]$, which is also known as the restriction point. At this checkpoint, cells decide whether to divide, delay the division, or enter a resting phase to repair the damaged DNA. The major components of this checkpoint are p16 and p21 as CKIs; CyclinD and CyclinE as cyclins; CDK4, CDK6, and CDK2 as CDKs. In addition, the proteins Rb and E2F are key components providing the transition from $\mathrm{G} 1$ to $\mathrm{S}$ phase by being phosphorylated by CDK4/6 and CDK2 in sequential order. Figure 1 is an illustration for this checkpoint, which was adapted from [31].

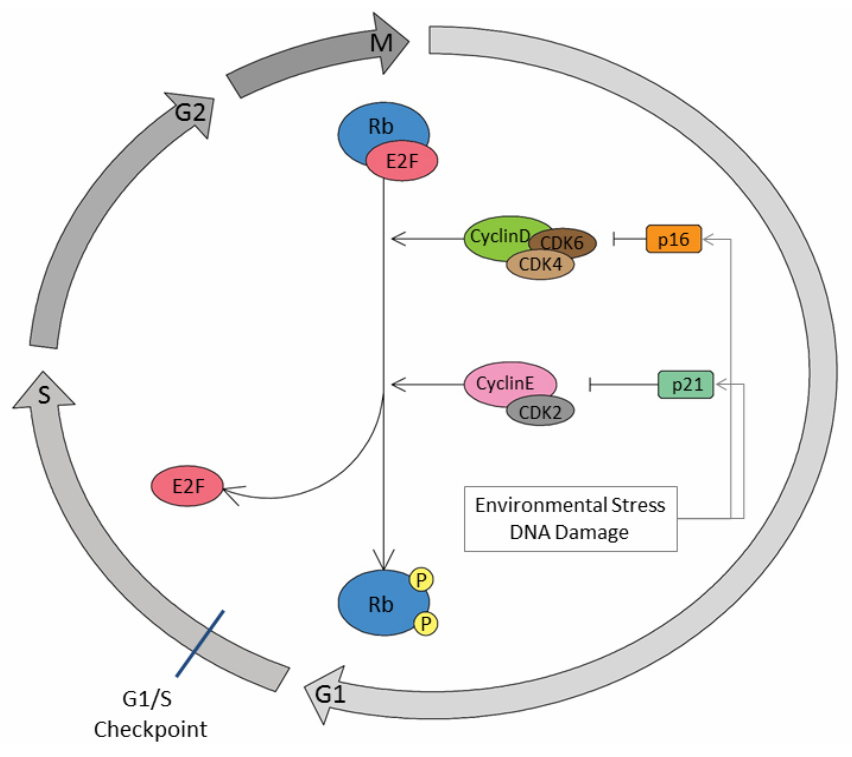

Figure 1

Schematic illustration of major events at G1/S checkpoint

In normal cells without DNA damage, the binding of CyclinD and CDK4/6 is established, which causes the initial phosphorylation of the Rb-E2F complex by CDK4/6. Phosphorylation of Rb-E2F complex results in the expression of CyclinE, which binds to CDK2 for second phosphorylation of Rb-E2F complex by which E2F is released from this complex. Release of E2F leads to the expression of genes necessary for the S phase implying G1/S checkpoint transition is fulfilled.

When DNA damage occurs, it is detected by CKIs p16 and p21. In the case where either of the p16 or p21 is mutated then the other member will be able to compensate for the loss of the dysfunctional CKI. When both p16 and p21 are mutated, they will not be able to perform their inhibitory functions. In this case, CKIs will be unable to block the phosphorylating abilities of CDKs, and the G1/S checkpoint is going to be passed by carrying DNA damage to the next phases of 
the cell cycle, which very likely will cause tumor formation after successive divisions.

\section{Reaction Systems}

In this section, we make use of RSs by considering its basic notions from [11, 12, 32] to model biological signaling pathways. The theory of RSs is explained by the following definitions.

Definition 1. Let a finite set $S$ of (biochemical or artificial) objects be given. A reaction is a triplet $r=(R, I, P)$ where $R, I$, and $P$ are finite nonempty sets with the condition $R \cap I=\emptyset$. If $S$ is a set such that $R, I, P \subseteq S$, then $r$ is a reaction in $S$.

The sets $R, I$, and $P$ in Definition 1 can also be written as $R_{i}, I_{i}$, and $P_{i}$, and they stand for the reactant set, the inhibitor set, and the product set of a reaction $r_{i}$, respectively. In addition, the set of all reactions in $S$ is denoted by $\operatorname{rac}(S)$ where $R_{i}, I_{i}, P_{i} \subseteq S$ for each $r_{i} \in \operatorname{rac}(S)$.

The definition above of an RS is originally designated by Ehrenfeucht and Rozenberg [12] to model biochemical reactions. According to the original definition given above, the set of inhibitors is required to be nonempty. However, our study concentrates on reactions in the cell where the objects are proteins. Different than biochemical reactions, the existence of inhibitors in protein-protein interactions is rare. For this reason, we do not consider the condition that the inhibitor set should be nonempty. This artificial condition could be fulfilled by introducing artificial inhibitor(s) to the system, but our consideration is more intuitive in the sense that readers who are not familiar with that type of models will not be confused if a reaction is never inhibited by any real inhibitors.

Definition 2. Let $T \subseteq S$ be a finite set.

(1) Let $r$ be a reaction. Then $r$ is enabled by $T$, denoted by $e n_{r}(T)$, if $R_{r} \subseteq T$ and $I_{r} \cap T=\varnothing$. The result of $r$ on $T$, denoted by $\operatorname{res}_{r}(T)=P_{r}$ if $e n_{r}(T)$, and $\operatorname{res}_{r}(T)=\emptyset$ otherwise.

(2) Let $A$ be a finite set of reactions. The result of $A$ on $T$, denoted by $\operatorname{res}_{A}(T)$, is defined by: $\operatorname{res}_{A}(T)=\cup_{r \in A}\left\{\operatorname{res}_{r}(T) \mid e n_{r}(T)\right\}$.

The set $T$ in Definition 2 stands for a state of a biochemical system, which consists of biochemical entities present in the current system. Therefore, a reaction $r$ is enabled by $T$ if $T$ includes all reactants of $r(R r \subseteq T)$ and it does not include any inhibitors of $r\left(I_{r} \cap T=\varnothing\right)$. For a set of reactions $A$, the result of $T$ on $A$ should be considered as cumulative, which means that the result of all reactions in the set $A$ is the union of results of all individual reactions in $A$. It means that, in fact, the execution of the reactions is applied in a parallel manner. 
Also, by definition of RSs, there is no conflict of resources. For example, if $r, s \in A$ are both enabled by $T$ and $R_{r} \cap R_{s} \neq \emptyset$ we still have $P_{r} \subseteq \operatorname{res}_{A}(T)$ and $P_{S} \subseteq \operatorname{res}_{A}(T)$. Thus, the set of results includes the products from both of the enabled reactions. This rule exhibits the threshold assumption in RSs: Either a resource is present in the system in a sufficient amount, or it is not present at all [32]. Therefore, the amount of objects is not counted in RSs, they are either present with a sufficient amount or absent. Hence, when modeling via RSs, we deal with not a quantitative, but a qualitative (binary) approach.

In our work, we slightly modify Definition 2 as follows:

Definition 3. Let $T \subseteq S$ be a finite set.

(1) Let $r$ be a reaction. Then $r$ is enabled by $T$, denoted by $e n_{r}(T)$, if $R_{r} \subseteq T$ and $I_{r} \nsubseteq \mathrm{T}$. The result of $r$ on $T$, denoted by $\operatorname{res}_{r}(T)=P_{r}$ if $e n_{r}(T)$, and $\operatorname{res}_{r}(T)=\emptyset$ otherwise.

(2) Let $A$ be a finite set of reactions. The result of $A$ on $T$, denoted by $\operatorname{res}_{A}(T)$, is defined by: $\operatorname{res}_{A}(T)=\cup_{r \in A}\left\{\operatorname{res}_{r}(T) \mid e n_{r}(T)\right\}$.

When modeling biological signaling pathways by defining the system via a set of reactions, it is noticed that all of the inhibitors in the set $I_{r}$ should be present to prevent the enabling of that reaction. Thus, we consider AND condition for inhibitors instead of the original OR condition. This modification is reflected to the definition by changing the condition $I_{r} \cap T \neq \varnothing$ in part (1) of the definition. Notice that by Definition 2 the presence of any object from the inhibitors set $I_{r}$ inhibits $r$, while by Definition 3 the presence of the whole set $I_{r}$ is needed to inhibit the reaction $r$. This new definition allows us to make our system very compact.

Definition 4. A Reaction System (RS) is an ordered pair $\mathcal{A}=(S, A)$ such that $S$ is a finite set and $A \subseteq \operatorname{rac}(S)$.

The set $S$ which is called the background set of $\mathcal{A}$, includes elements called entities to represent molecular objects such as atoms, molecules, proteins, and/or artificial (technical) objects that may be present in the states of the system modeled by $\mathcal{A}$. The set $A$ is called the set of reactions of $\mathcal{A}$.

The following interactive process is defined to formalize the dynamic behavior of an RS:

Definition 5. Let $\mathcal{A}=(S, A)$ be an RS and let $n \geq 0$ be an integer. An n-step interactive process in $\mathcal{A}$ is a pair $\pi=(\gamma, \delta)$ of finite sequences such that $\gamma=C_{0}, C_{1}, \ldots, C_{n}$ and $\delta=D_{0}, D_{1}, \ldots, D_{n}$ where $C_{0}, \ldots, C_{n}, D_{0}, \ldots, D_{n} \subseteq S, D_{0}=\emptyset$, and $D_{i}=\operatorname{res}_{\mathcal{A}}\left(D_{i-1} \cup C_{i-1}\right)$ for all $i \in\{1, \ldots, n\}$.

In each step of a simulation, all enabled reactions are applied in parallel, and the whole system is deterministic. 


\section{Modeling and Simulations}

\subsection{Model Construction}

The RS for modeling the G1/S checkpoint of the cell cycle is created by considering the major components in this specific cell cycle checkpoint which is mentioned in Subsection 2.3.1. Reactions are written by using the theory of RSs explained in Section 3. The system is defined as follows.

$S=S_{0} \cup S_{1}$ with $S_{0}=\{P, S P h, A, p 16$ mutator, $p 21$ mutator $\}$ where $P$ is Phosphate, $S P h$ is S Phase Transfer, $A$ is an aberrancy in the G1 phase such as DNA damage, mutations (except p16 or p21 mutations) or another dysfunction in the system, $p 16$ mutator and $p 21$ mutator are causes for mutations on $\mathrm{p} 16$ and p21 respectively. $S_{1}$ contains proteins: $S_{1}=\left\{A_{i j} \mid i \in\{C D K 4 / 6, C y c D, C D K 2\right.$, $C y c E, R b, E 2 F, R b / E 2 F, C 1, C 2, p 16, p 21\}$ and $j \in\{n, h p, f, m\}\}$ with $C 1$ being the CycD-CDK4/6 complex and $C 2$ being the $\mathrm{CycE}-\mathrm{CDK} 2$ complex; and $n, h p, f$, and $m$ representing non-expressed, hypo-phosphorylated, fully expressed and mutated proteins, respectively.

The 23 reactions given below represent the G1/S checkpoint of the cell cycle.

$$
\begin{aligned}
& r_{1}=\left(\left\{A_{C D K 4 / 6, f}, A_{C y c D, f}\right\},\left\{A, A_{p 16, f}\right\},\left\{A_{C D K 4 / 6, f}, A_{C y c D, f}, A_{C 1, f}\right\}\right) \\
& r_{2}=\left(\left\{A_{C 1, f}, A_{R b / E 2 F, f}, P\right\}, \emptyset,\left\{A_{C 1, f}, A_{R b / E 2 F, h p}\right\}\right) \\
& r_{3}=\left(\left\{A_{R b / E 2 F, h p}, A_{C y c E, n}\right\}, \emptyset,\left\{A_{R b / E 2 F, h p}, A_{C y c E, f}\right\}\right) \\
& r_{4}=\left(\left\{A_{C D K 2, f}, A_{C y c E, f}\right\},\left\{A, A_{p 21, f}\right\},\left\{A_{C D K 2, f}, A_{C y c E, f}, A_{C 2, f}\right\}\right) \\
& r_{5}=\left(\left\{A_{C 2, f}, A_{R b / E 2 F, h p}, P\right\}, \emptyset,\left\{A_{C 2, f}, A_{R b, f}, A_{E 2 F, f}\right\}\right) \\
& r_{6}=\left(\left\{A_{E 2 F, f}\right\}, \emptyset,\{S P h\}\right) \\
& r_{7}=(\{P\}, \emptyset,\{P\}) \\
& r_{8}=(\{A\}, \emptyset,\{A\}) \\
& r_{9}=\left(\left\{A_{C D K 2, f}\right\}, \emptyset,\left\{A_{C D K 2, f}\right\}\right) \\
& r_{10}=\left(\left\{A_{C D K 4 / 6, f}\right\}, \emptyset,\left\{A_{C D K 4 / 6, f}\right\}\right) \\
& r_{11}=\left(\left\{A_{C y c D, f}\right\}, \emptyset,\left\{A_{C y c D, f}\right\}\right) \\
& r_{12}=\left(\left\{A_{C y c E, f}\right\}, \emptyset,\left\{A_{C y c E, f}\right\}\right) \\
& r_{13}=\left(\left\{A_{R b, f}\right\}, \emptyset,\left\{A_{R b, f}\right\}\right) \\
& r_{14}=\left(\left\{A_{E 2 F, f}\right\}, \emptyset,\left\{A_{E 2 F, f}\right\}\right) \\
& r_{15}=\left(\left\{A_{p 16, m}\right\}, \emptyset,\left\{A_{p 16, m}\right\}\right)
\end{aligned}
$$




$$
\begin{aligned}
& r_{16}=\left(\left\{A_{p 21, m}\right\}, \emptyset,\left\{A_{p 21, m}\right\}\right) \\
& r_{17}=\left(\left\{A_{p 16, f}, p 1 \text { mutator }\right\}, \emptyset,\left\{A_{p 16, m}\right\}\right) \\
& r_{18}=\left(\left\{A_{p 21, f}, p 21 \text { mutator }\right\}, \emptyset,\left\{A_{p 21, m}\right\}\right) \\
& r_{19}=\left(\left\{A_{p 16, f}\right\},\{\text { p16mutator }\},\left\{A_{p 16, f}\right\}\right) \\
& r_{20}=\left(\left\{A_{p 21, f}\right\},\{p 21 \text { mutator }\},\left\{A_{p 21, f}\right\}\right) \\
& r_{21}=\left(\left\{A_{C y C E, n}\right\},\left\{A_{R b / E 2 F, h p}\right\},\left\{A_{C y c E, n}\right\}\right) \\
& r_{22}=\left(\left\{A_{R b / E 2 F, f}\right\},\left\{A_{C 1, f}, P\right\},\left\{A_{R b / E 2 F, f}\right\}\right) \\
& r_{23}=\left(\left\{A_{R b / E 2 F, h p}\right\},\left\{A_{C 2, f}, P\right\},\left\{A_{R b / E 2 F, h p}\right\}\right)
\end{aligned}
$$

The first six reactions illustrate the main biological phenomena happening during the G1 phase of the cell cycle, as explained in Subsection 2.3.1. Reaction $r_{1}$ represents the binding of CyclinD and CDK4/6 complex. This reaction goes in the presence of fully expressed CDK4/6 and fully expressed CyclinD, and in the absence of any dysfunction and fully expressed p16. As products of $r_{1}$, we obtain fully expressed CDK4/6, fully expressed CyclinD, and fully expressed C1 (CyclinD-CDK4/6 complex).

Similarly, $r_{2}$ represents the hypo-phosphorylation of $\mathrm{Rb}$ by the CyclinD CDK4/6 complex, while $r_{3}$ stands for the expression of CyclinE by the existence of hypophosphorylated Rb. In $r_{4}$, the formation of the CyclinE-CDK2 complex with the conditions of having no dysfunction and having no fully expressed $\mathrm{p} 21$ is presented. Reaction $r_{5}$ represents the hyper-phosphorylation of $\mathrm{Rb}$ to cause the release of E2F from the $\mathrm{Rb}-\mathrm{E} 2 \mathrm{~F}$ complex by the existence of the CyclinE CDK2 complex. Lastly in $r_{6}$, the transition from $\mathrm{G} 1$ to $\mathrm{S}$ checkpoint is illustrated.

Reactions through $r_{7}$ to $r_{16}$ are stated to keep the reactants of these reactions into the system. For example, phosphate is always available in the cell via the existence of ATP [33], and it won't be vanished or disappear after some processes occur. Reaction $r_{7}$ helps us to keep phosphate in the system. Reactions $r_{17}$ and $r_{18}$ illustrate the mutations on CKIs p16 and p21, respectively, while reactions $r_{19}$ and $r_{20}$ are stated to keep fully expressed p16 and p21 in the system when there is nothing in the cell to cause their mutations. Non-expressed CyclinE remains nonexpressed in the system as long as the $\mathrm{Rb}-\mathrm{E} 2 \mathrm{~F}$ complex is not hypophosphorylated, which is presented in $r_{21}$. Similarly, in $r_{22}$ and $r_{23}$, fully expressed and hypo-phosphorylated $\mathrm{Rb}-\mathrm{E} 2 \mathrm{~F}$ complexes are preserved as there is no fully expressed $\mathrm{C} 1$ and phosphate, and no fully expressed $\mathrm{C} 2$ and phosphate are present respectively. 


\subsection{Simulations and Validation of the Model}

In order to conduct simulations for different scenarios, a $\mathrm{C}++$ program is written in which we define Boolean variables for each component in $S$. We start with setting all components to false, and then we introduce the initial set for stating the existing components at the beginning of the simulation. We also check if there is a mutation on tumor suppressor genes p16 and p21 interactively. Also, the existence of an aberrancy in G1 is set to true or false, in a similar way.

After the initialization is completed for each scenario, implementation of the reactions is realized within a loop structure in which several rounds are run to obtain the final result. Within each round, we first check which reactions can be implemented based on the currently existing components. After recording the enabled reactions, we set all components to false to have only those which are produced right after the enabled reactions run. Then this product set becomes the initial set of the next round, and the same procedure is repeated until our stopping criteria $\mathrm{SPh}=1$ is achieved, which means that the G1-S checkpoint is passed. If the stopping criterion is not attained, but we obtain an infinite loop, then we understand that $\mathrm{G} 1$ to $\mathrm{S}$ transition is not achieved. In each scenario, we consider the CDK4/6 complex, CyclinD, CDK2, and the Rb/E2F complex being fully expressed. The existence of aberrancy in G1 and mutations on p16 and p21 are interactively examined at the beginning of each simulation. Also, it is possible to introduce different states for the existing components in the system between each round of simulations, which is very important for deducing model predictions.

In order to validate our model, scenarios considered in our simulations are as follows:

\subsubsection{Normal(wild-type) Cell - p16 and p21 Non-mutated, no Aberrancy in G1 is Present}

We first consider the scenario in which the tumor suppressors p16 and p21 are non-mutated, and there is no aberrancy in the G1 phase. The expected consequence of this scenario is the transition from G1 to $\mathrm{S}$ phase, which is confirmed by our simulation as follows.

Components that are initially present are $\mathrm{P}, \mathrm{A}_{\mathrm{CDK} 4 / 6, \mathrm{f}}, \mathrm{A}_{\mathrm{CycD}, \mathrm{f}}, \mathrm{A}_{\mathrm{CDK} 2, \mathrm{f}}, \mathrm{A}_{\mathrm{CycE}, \mathrm{n}}$, $\mathrm{A}_{\mathrm{Rb} / \mathrm{E} 2 \mathrm{~F}, \mathrm{f}}, \mathrm{A}_{\mathrm{p} 16, \mathrm{f}}, \mathrm{A}_{\mathrm{p} 21, \mathrm{f}}$. The first round of the simulation starts with checking the definition of the first reaction $r_{1}$. It can be seen that $r_{1}$ is enabled due to the existence of $\mathrm{A}_{\mathrm{CDK} 4 / 6, \mathrm{f}}, \mathrm{A}_{\mathrm{CycD}, \mathrm{f}}$ as reactants, and non-existence of $A$ among the inhibitors. Similarly, based on the definition of $r_{2}$, nonexistence of $A_{C 1, f}$ among the reactants causes $r_{2}$ not to be enabled. The first round of the simulation for this scenario is fully explained in Table 1 , where the enabled reactions are run in parallel, and the products set of the first round is obtained as $\left\{\boldsymbol{A}_{C D K 4 / 6, f}, \boldsymbol{A}_{C y C D, f}, \boldsymbol{A}_{C 1, f}, \boldsymbol{P}, \boldsymbol{A}_{C D K 2, f}, \boldsymbol{A}_{p 16, f}, \boldsymbol{A}_{p 21, f}, \boldsymbol{A}_{C y C E, n}, \boldsymbol{A}_{\boldsymbol{R} b / E 2 F, f}\right\}$ which is at the same time the initial components set of the second round of the simulation. 
Table 1

Details of the first round of the simulation for a normal (wild-type) cell., where the initial components are: $\mathrm{P}, \mathrm{A}_{\mathrm{CDK} 4 / 6, \mathrm{f}}, \mathrm{A}_{\mathrm{CycD}, \mathrm{f}}, \mathrm{A}_{\mathrm{CDK} 2, \mathrm{f}}, \mathrm{A}_{\mathrm{CycE}, \mathrm{n}}, \mathrm{A}_{\mathrm{Rb} / \mathrm{E} 2 \mathrm{~F}, \mathrm{f}}, \mathrm{A}_{\mathrm{p} 16, \mathrm{f}}, \mathrm{A}_{\mathrm{p} 21, \mathrm{f}}$ and available components are marked in bold.

\begin{tabular}{|c|c|c|c|c|}
\hline Reactio & Reactants & Inhibitors & status & Products \\
\hline$r_{1}$ & $\left\{A_{C D K 4 / 6, f}, A_{C y c D, f}\right\}$ & $\left\{A, A_{p 16, f}\right\}$ & enable & $\left\{A_{C D K 4 / 6, f}, A_{C y c D, f}, A_{C 1}\right.$ \\
\hline$r_{2}$ & $\left\{A_{C 1, f}, \boldsymbol{A}_{\boldsymbol{R} \boldsymbol{B} / \mathbf{E} 2 F, f}, \boldsymbol{P}\right\}$ & $\varnothing$ & disable & - \\
\hline$r_{3}$ & $\left\{A_{R b / E 2 F, h p}, \boldsymbol{A}_{\boldsymbol{C y c E}, \boldsymbol{n}}\right\}$ & $\varnothing$ & disable & - \\
\hline$r_{4}$ & $\left\{\boldsymbol{A}_{C D K 2, f}, A_{C y c E, f}\right\}$ & $\left\{A, A_{p 21, f}\right\}$ & disable & - \\
\hline$r_{5}$ & $\left\{A_{C 2, f}, A_{R b / E 2 F, h p}, \mathbf{P}\right.$ & $\varnothing$ & disable & - \\
\hline$r_{6}$ & $\left\{A_{E 2 F, f}\right\}$ & $\varnothing$ & disable & - \\
\hline$r_{7}$ & $\{\boldsymbol{P}\}$ & $\varnothing$ & enable & $\{\boldsymbol{P}\}$ \\
\hline$r_{8}$ & $\{A\}$ & $\varnothing$ & disable & - \\
\hline$r_{9}$ & $\left\{A_{C D K 2, f}\right\}$ & $\varnothing$ & enable & $\left\{A_{C D K 2, f}\right\}$ \\
\hline$r_{10}$ & $\left\{A_{C D K 4 / 6, f}\right\}$ & $\varnothing$ & enable & $\left\{A_{C D K 4 / 6, f}\right\}$ \\
\hline$r_{11}$ & $\left\{\boldsymbol{A}_{C y c D, f}\right\}$ & $\varnothing$ & enable & $\left\{\boldsymbol{A}_{\boldsymbol{C y c D}, f}\right\}$ \\
\hline$r_{12}$ & $\left\{A_{C y c E, f}\right\}$ & $\varnothing$ & disable & - \\
\hline$r_{13}$ & $\left\{A_{R b, f}\right\}$ & $\varnothing$ & disable & - \\
\hline$r_{14}$ & $\left\{A_{E 2 F, f}\right\}$ & $\varnothing$ & disable & - \\
\hline$r_{15}$ & $\left\{A_{p 16, m}\right\}$ & $\varnothing$ & disable & - \\
\hline$r_{16}$ & $\left\{A_{p 21, m}\right\}$ & $\varnothing$ & disable & - \\
\hline$r_{17}$ & $\left\{\boldsymbol{A}_{p 16, f}, p 16\right.$ mutator & $\varnothing$ & disable & - \\
\hline$r_{18}$ & $\left\{\boldsymbol{A}_{\boldsymbol{p 2 1}, f}, p 21\right.$ mutator & $\varnothing$ & disable & - \\
\hline$r_{19}$ & $\left\{\boldsymbol{A}_{\boldsymbol{p 1 6}, f}\right\}$ & $\{p 16$ mutator $\}$ & enable & $\left\{\boldsymbol{A}_{\boldsymbol{p 1 6}, f}\right\}$ \\
\hline$r_{20}$ & $\left\{A_{p 21, f}\right\}$ & $\{p 21$ mutator $\}$ & enable & $\left\{A_{p 21, f}\right\}$ \\
\hline$r_{21}$ & $\left\{\boldsymbol{A}_{\boldsymbol{C y c E}, \boldsymbol{n}}\right\}$ & $\left\{A_{R b / E 2 F, h p}\right\}$ & enable & $\left\{A_{C y c E, n}\right\}$ \\
\hline$r_{22}$ & $\left\{A_{R b / E 2 F, f}\right\}$ & $\left\{A_{C 1, f}, \boldsymbol{P}\right\}$ & enable & $\left\{A_{R b / E 2 F, f}\right\}$ \\
\hline$r_{23}$ & $\left\{A_{R b / E 2 F, h p}\right\}$ & $\left\{A_{C 2, f}, \boldsymbol{P}\right\}$ & disable & - \\
\hline
\end{tabular}

With the same understanding, rounds are run until there is no difference between the initial components set and the product set of a round. For this specific scenario, it occurred at the end of the sixth round, and the following components are obtained as the products of the simulation: $\mathrm{SPh}, \mathrm{P}, \mathrm{A}_{\mathrm{CDK} 4 / 6, \mathrm{f}}, \mathrm{A}_{\mathrm{CycD}, \mathrm{f}}, \mathrm{A}_{\mathrm{CDK} 2, \mathrm{f}}$, $A_{\mathrm{CycE}, \mathrm{f}}, A_{\mathrm{Rb}, \mathrm{f}}, \mathrm{A}_{\mathrm{E} 2 \mathrm{~F}, \mathrm{f}}, \mathrm{A}_{\mathrm{C} 1, \mathrm{f}}, \mathrm{A}_{\mathrm{C} 2 \mathrm{f}}, \mathrm{A}_{\mathrm{p} 16, \mathrm{f}}, \mathrm{A}_{\mathrm{p} 21, \mathrm{f}}$, where $\mathrm{G} 1$ to $\mathrm{S}$ transition is realized by the existence of $A_{R b, f}$ in the products set. The detailed illustration of the simulation for this specific scenario is given in Figure 2. 


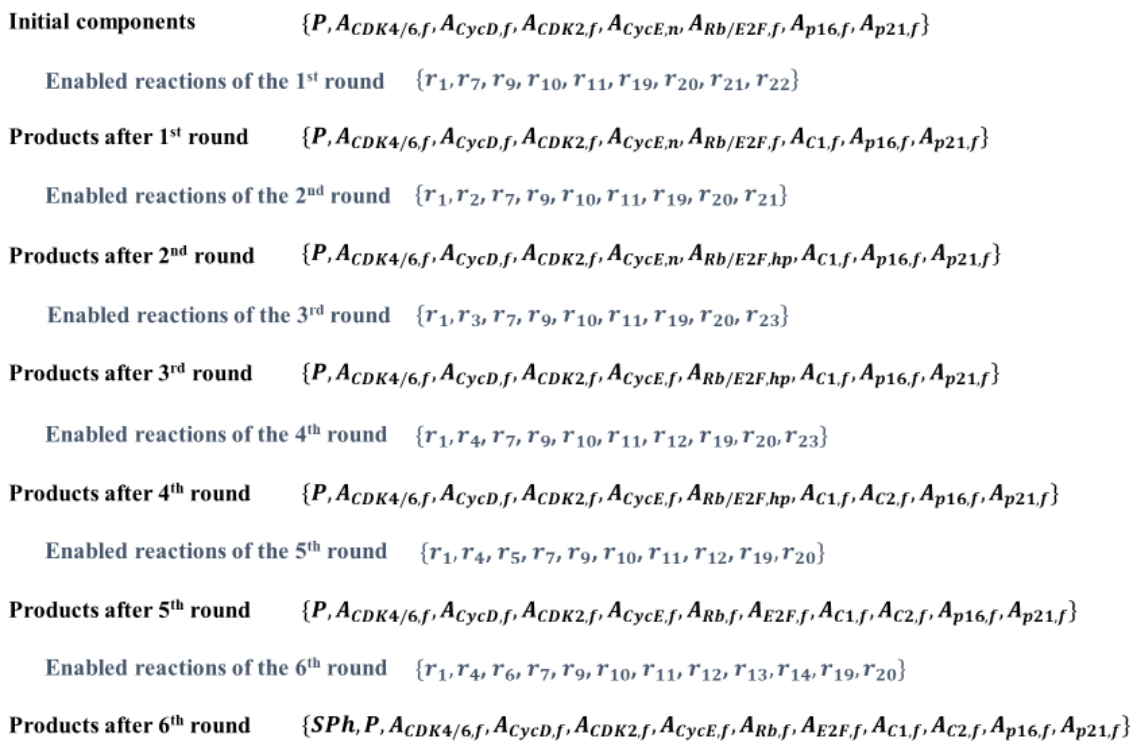

Figure 2

Details of the simulation for a wild type cell

\subsubsection{Aberrancy in G1 in the Absence of p16 and p21 Mutations}

In the second scenario, it is considered that there is an aberrancy in the G1 phase, but both p16 and p21 are functional. As expected, our simulation shows that G1/S transition is not realized since p16 blocks the formation of the CyclinD-CDK4/6 complex to prevent the checkpoint to be passed.

In our RS, the initial components are: $\mathrm{P}, \mathrm{A}, \mathrm{A}_{\mathrm{CDK} 4 / 6, \mathrm{f}}, \mathrm{A}_{\mathrm{CycD}, \mathrm{f}}, \mathrm{A}_{\mathrm{CDK} 2, \mathrm{f}}, \mathrm{A}_{\mathrm{CycE}, \mathrm{n}}$, $A_{\mathrm{Rb} / \mathrm{E} 2 \mathrm{~F}, \mathrm{f}}, \mathrm{A}_{\mathrm{p} 16, \mathrm{f}}, \mathrm{A}_{\mathrm{p} 21, \mathrm{f}} . \mathrm{A}$ loop structure is obtained by the conducted simulation, which reflected no change on these components even in the first round.

\subsubsection{Aberrancy in G1 when p16 is Mutated, but p21 is Functional}

The third scenario assumes that there is an aberrancy in the G1 phase, p16 is mutated, and p21 is functional. In this case, p16 will not be able to block the formation of the CyclinD-CDK4/6 complex, and $\mathrm{Rb} / \mathrm{E} 2 \mathrm{~F}$ will be hypophosphorylated to cause the transcription of CyclinE. However, since p21 is functional, it will block the formation of the CyclinE-CDK2 complex, and thus, G1/S transition will be blocked as our simulation results reflected.

For this scenario, initial components in the RS are $\mathrm{P}, \mathrm{A}, \mathrm{A}_{\mathrm{CDK} 4 / 6, \mathrm{f}}, \mathrm{A}_{\mathrm{CycD}, \mathrm{f}}, \mathrm{A}_{\mathrm{CDK} 2, \mathrm{f}}$, $A_{C y c E, n}, A_{R b / E 2 F, f}, A_{p 16, m}, A_{p 21, f}$. After four rounds, we obtain a loop structure with the components $\mathrm{P}, \mathrm{A}, \mathrm{p} 16$ mutator, $\mathrm{A}_{\mathrm{CDK} 4 / 6, \mathrm{f}}, \mathrm{A}_{\mathrm{CyCD}, \mathrm{f}}, \mathrm{A}_{\mathrm{CDK} 2, \mathrm{f}}, \mathrm{A}_{\mathrm{CycE}, \mathrm{f}}, \mathrm{A}_{\mathrm{Rb} / \mathrm{E} 2 \mathrm{~F}, \mathrm{hp}}$, $A_{C 1, f}, A_{p 16, m}, A_{p 21, f}$ meaning that G1/S transition is not achieved (Figure 3). 


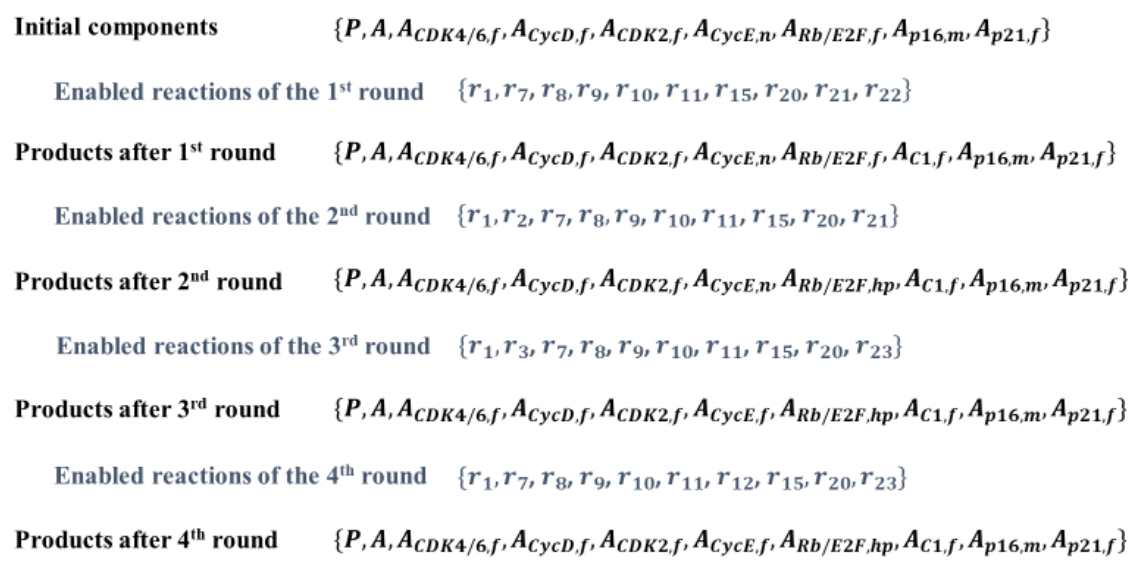

Figure 3

Simulation details for the scenario where there is an aberrancy in G1 when p16 is mutated, but p21 is functional

\subsubsection{Aberrancy in G1 when p21 is Mutated, but p16 is Functional}

In the fourth scenario, we consider that there is an aberrancy in the G1 phase, p21 is mutated, and p16 is functional. Even though the CKI p21 is mutated, in the first part of the pathway, functional p16 will prevent the hypo-phosphorylation of $\mathrm{Rb} / \mathrm{E} 2 \mathrm{~F}$ complex, and thus the G1/S transition will be blocked, as we have obtained by our simulation results on this scenario:

The initial components of this scenario are $\mathrm{P}, \mathrm{A}, \mathrm{A}_{\mathrm{CDK} 4 / 6, \mathrm{f}}, \mathrm{A}_{\mathrm{CycD}, \mathrm{f}}, \mathrm{A}_{\mathrm{CDK} 2, \mathrm{f}}, \mathrm{A}_{\mathrm{CycE}, \mathrm{n}}$, $\mathrm{A}_{\mathrm{Rb} / \mathrm{E} 2 \mathrm{~F}, \mathrm{f}}, \mathrm{A}_{\mathrm{p} 16, \mathrm{f}}, \mathrm{A}_{\mathrm{p} 21, \mathrm{~m}}$. As a result, $r_{7}, r_{8}, r_{9}, r_{10}, r_{11}, r_{16}, r_{19}, r_{21}, r_{22}$ are executed in the first round of the simulation; however, the components in the simulation did not change, and the checkpoint is not passed.

\subsubsection{Aberrancy in G1 in the Presence of p16 and p21 Mutations}

Our last scenario considers the worst case, in which there is an aberrancy in the G1 phase, and both p16 and p21 are mutated. In such a case, there will be no functional CKI that can block neither the hypo-phosphorylation nor the full phosphorylation of the $\mathrm{Rb} / \mathrm{E} 2 \mathrm{~F}$ complex. Our simulation shows that the G1/S transition cannot be blocked in this case.

The initial components for the simulation of this scenario are: $\mathrm{P}, \mathrm{A}, \mathrm{A}_{\mathrm{CDK} 4 / 6, \mathrm{f}}$, $A_{C y c D, f}, A_{C D K 2, f}, A_{C y c E, n}, A_{R b / E 2 F, f}, A_{p 16, m}, A_{p 21, m}$. The obtained simulation results reflected that the stopping criteria $\mathrm{SPh}$ is attained within six rounds. The resulting components are as follows: $\mathrm{SPh}, \mathrm{P}, \mathrm{A}, \mathrm{A}_{\mathrm{CDK} 4 / 6, \mathrm{f}}, \mathrm{A}_{\mathrm{CycD}, \mathrm{f}}, \mathrm{A}_{\mathrm{CDK} 2, \mathrm{f}}, \mathrm{A}_{\mathrm{CycE}, \mathrm{f}}, \mathrm{A}_{\mathrm{Rb}, \mathrm{f}}$, $A_{E 2 F, f}, A_{C 1, f}, A_{C 2, f}, A_{p 16, m}, A_{p 21, m}$ which can be seen in Figure 4. 


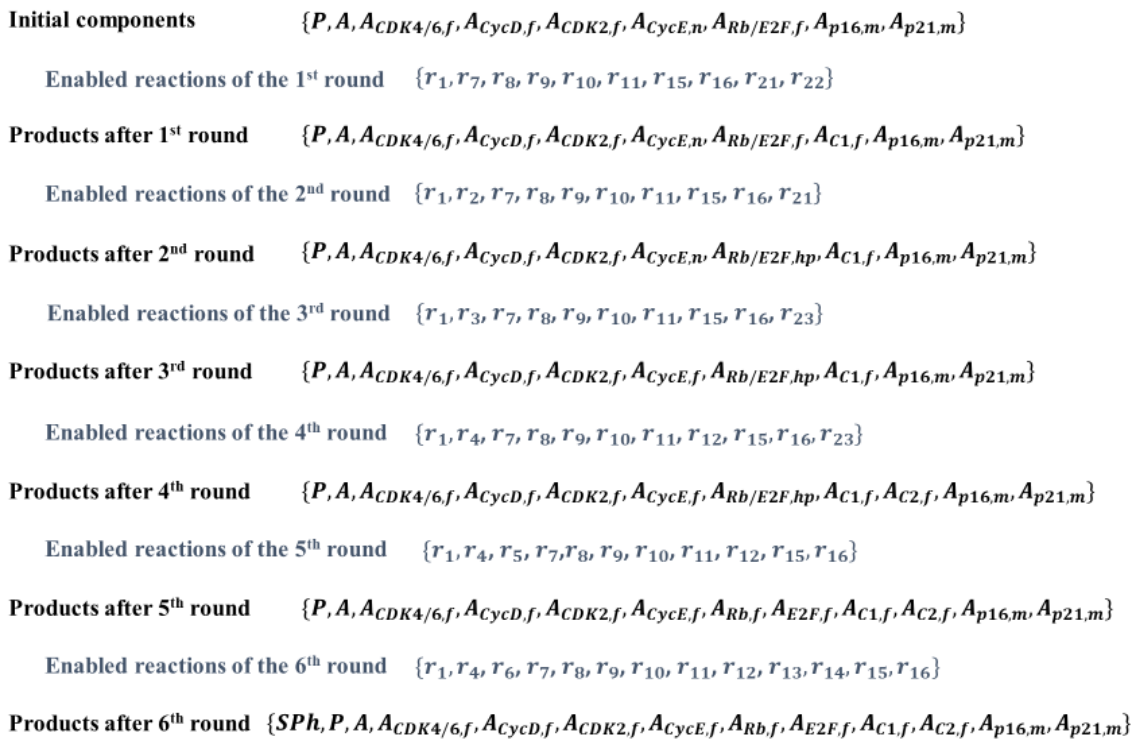

Figure 4

Simulation details for the scenario where there is an aberrancy in G1 and both p16 and p21 are mutated

As can be seen in the above scenarios, the conducted simulations have worked well and provided the desired outcomes. Other than these five scenarios, it is possible to conduct simulations for various possible scenarios with additional biological conditions to find potential results prior to wet lab experiments.

\section{Comparison with Other Modeling Techniques}

As different approaches to mathematically model biological systems have their own advantages and disadvantages; modeling with RSs also has its own strengths and weaknesses. Having a deterministic modeling approach; it provides a compact, simple, and fast modeling and simulation of such systems. One may not need any specific software package to run simulations, since it is relatively easy to program a reaction system. Also, much larger systems can be created by RSs with less computing effort than hybrid and stochastic modeling approaches. RSs also able to simulate interactive processes when after each round some objects may be added to the system. Actually, for large size reaction systems there exists also a software package helping to do simulations [34]. Meanwhile; not being able to represent non-deterministic or quantitative systems and the various strength or speed of the reactions might be considered weaknesses of modeling with RSs. 


\section{Conclusions and Further Work}

In this paper, we have implemented the notion of RSs for modeling the G1/S checkpoint of the human cell cycle, which has importance in tumor formation.

The proposed model is proven to be efficacious and biologically relevant by obtaining expected results from simulations, for some specific scenarios. Thus, this qualitative RSs model is able to simulate various scenarios related to the G1/S checkpoint of the cell cycle in a simple, elegant, and correct manner. We show that modeling using RSs, helps us to observe biochemical reactions in a compact way. In addition, the constructed RS can simulate various scenarios, which could help to find biological results in-silico. In-silico studies can help describe potential candidates for drug signature identification. There are many possibilities of mathematical modeling of biological systems. We introduce here, a novel RSs methodology that can be used in the search for drug signature identification. Moreover, simulations can be helpful for drug design, by attaching biological components (e.g. inhibitors) to the reactions in the system.

As future work, our plan is to extend the RS of the whole human cell cycle, by additionally considering the G2/M checkpoint and the spindle checkpoint in the $\mathrm{M}$ phase of mitosis.

\section{Acknowledgement}

We thank Prof. Dr. Rza Bashirov for his encouragement and support on this study. Also, we acknowledge Dr. Mani Mehraei for his help and useful comments on this research. Comments of the anonymous reviewers are gratefully acknowledged.

\section{References}

[1] Bartocci, E., \& Lió, P. (2016) Computational modeling, formal analysis, and tools for systems biology. PLoS computational biology, 12(1), e1004591

[2] Tyson, J. J. (1991) Modeling the cell division cycle: cdc2 and cyclin interactions. Proceedings of the National Academy of Sciences, 88(16), 7328-7332

[3] Aguda, B. D. (1999) A quantitative analysis of the kinetics of the G2 DNA damage checkpoint system. Proceedings of the National Academy of Sciences, 96(20), 11352-11357

[4] Haberichter, T., Mädge, B., Christopher, R. A., Yoshioka, N., Dhiman, A., Miller, R., ... \& Dowdy, S. F. (2007) A systems biology dynamical model of mammalian G1 cell cycle progression. Molecular systems biology, 3(1), 84

[5] Conradie, R., Bruggeman, F. J., Ciliberto, A., Csikász- Nagy, A., Novák, B., Westerhoff, H. V., \& Snoep, J. L. (2010) Restriction point control of the 
mammalian cell cycle via the cyclin E/Cdk2: p27 complex. The FEBS journal, 277(2), 357-367

[6] Herajy, M., Schwarick, M., \& Heiner, M. (2013) Hybrid Petri nets for modelling the eukaryotic cell cycle. In Transactions on Petri Nets and Other Models of Concurrency VIII (pp. 123-141) Springer, Berlin, Heidelberg

[7] Abroudi, A., Samarasinghe, S., \& Kulasiri, D. (2020) Towards abstraction of computational modelling of mammalian cell cycle: Model reduction pipeline incorporating multi-level hybrid petri nets. Journal of theoretical biology, 496, 110212

[8] Nagasaki, M., Matsuno, H., \& Miyano, S. (2006) Simulation-based validation of the p53 transcriptional activity with hybrid functional Petri net. In silico biology, 6(1, 2), 1-13

[9] Akçay, N. İ., Bashirov, R., \& Tüzmen, Ş. (2015) Validation of signalling pathways: Case study of the p16-mediated pathway. Journal of bioinformatics and computational biology, 13(02), 1550007

[10] Bashirov, R., \& Akçay, N. I. (2018) Stochastic Simulation-based Prediction of the Behavior of the p16-mediated Signaling Pathway. Fundamenta Informaticae, 160(1-2), 167-179

[11] Ehrenfeucht, A., \& Rozenberg, G. (2007) Events and modules in reaction systems. Theoretical Computer Science, 376(1-2), 3-16

[12] Ehrenfeucht, A., \& Rozenberg, G. (2004, December) Basic notions of reaction systems. In International Conference on Developments in Language Theory (pp. 27-29) Springer, Berlin, Heidelberg

[13] Azimi, S., Iancu, B., \& Petre, I. (2014) Reaction system models for the heat shock response. Fundamenta Informaticae, 131(3-4), 299-312

[14] Barbuti, R., Bove, P., Gori, R., Levi, F., \& Milazzo, P. (2018, September) Simulating Gene Regulatory Networks using Reaction Systems. In CS\&P (Vol. 2240)

[15] Mehraei, M., Nagy, B., Akcay, N. I., \& Tüzmen, Ş. (2019) Potential Therapeutic Modalities of Reawakening Fetal Hemoglobin Simulated by Reaction Systems. Acta Polytechnica Hungarica, 16(3)

[16] Vermeulen, K., Van Bockstaele, D. R., \& Berneman, Z. N. (2003) The cell cycle: a review of regulation, deregulation and therapeutic targets in cancer. Cell proliferation, 36(3), 131-149

[17] Lodish, H., Berk, A., Kaiser, C. A., Kaiser, C., Krieger, M., Scott, M. P., ... \& Matsudaira, P. (2008) Molecular cell biology. Macmillan

[18] De Souza, C. P., \& Osmani, S. A. (2007) Mitosis, not just open or closed. Eukaryotic cell, 6(9), 1521-1527 
[19] Karam, J. A. (2009) Apoptosis in carcinogenesis and chemotherapy. Netherlands: Springer

[20] Alberts, B., Johnson, A., Lewis, J., Raff, M., Roberts, K., \& Walter, P. (2008) Chapter 18 Apoptosis: programmed cell death eliminates unwanted cells. Molecular Biology of the Cell (Textbook) $5^{\text {th }}$ ed. New York: Garland Science, 1115

[21] Kerr, J. F. (1965) A histochemical study of hypertrophy and ischaemic injury of rat liver with special reference to changes in lysosomes. The Journal of pathology and bacteriology, 90(2), 419-435

[22] Lim, S., \& Kaldis, P. (2013) Cdks, cyclins and CKIs: roles beyond cell cycle regulation. Development, 140(15), 3079-3093

[23] Malumbres, M., \& Barbacid, M. (2009) Cell cycle, CDKs and cancer: a changing paradigm. Nature reviews cancer, 9(3), 153-166

[24] Kastan, M. B., \& Bartek, J. (2004) Cell-cycle checkpoints and cancer. Nature, 432(7015), 316-323

[25] Bertoli, C., Skotheim, J. M., \& De Bruin, R. A. (2013) Control of cell cycle transcription during $\mathrm{G} 1$ and $\mathrm{S}$ phases. Nature reviews Molecular cell biology, 14(8), 518-528

[26] Bartek, J., \& Lukas, J. (2001) Mammalian G1-and S-phase checkpoints in response to DNA damage. Current opinion in cell biology, 13(6), 738-747

[27] Wang, Y., Ji, P., Liu, J., Broaddus, R. R., Xue, F., \& Zhang, W. (2009) Centrosome-associated regulators of the G 2/M checkpoint as targets for cancer therapy. Molecular cancer, 8(1), 1-13

[28] Löbrich, M., \& Jeggo, P. A. (2007) The impact of a negligent G2/M checkpoint on genomic instability and cancer induction. Nature Reviews Cancer, 7(11), 861-869

[29] Peters, J. M. (1998) SCF and APC: the Yin and Yang of cell cycle regulated proteolysis. Current opinion in cell biology, 10(6), 759-768

[30] Ciosk, R., Zachariae, W., Michaelis, C., Shevchenko, A., Mann, M., \& Nasmyth, K. (1998) An ESP1/PDS1 complex regulates loss of sister chromatid cohesion at the metaphase to anaphase transition in yeast. Cell, 93(6), 1067-1076

[31] Coller, H. A. (2007) What's taking so long? S-phase entry from quiescence versus proliferation. Nature reviews Molecular cell biology, 8(8), 667-670

[32] Brijder, R., Ehrenfeucht, A., Main, M., \& Rozenberg, G. (2011) A tour of reaction systems. International Journal of Foundations of Computer Science, 22(07), 1499-1517 
[33] van Keulen, G., Siebring, J., \& Dijkhuizen, L. (2011) Central carbon metabolic pathways in Streptomyces. Streptomyces: Molecular biology and biotechnology, 105-124

[34] Ivanov, S., Rogojin, V., Azimi, S., \& Petre, I. (2018) Webrsim: A webbased reaction systems simulator. In Enjoying Natural Computing (pp. 170181) Springer, Cham 